\title{
New interactive solar flare modeling and advanced radio diagnostics tools
}

\author{
Gregory D. Fleishman ${ }^{1,2}$, Gelu M. Nita ${ }^{1}$ and Dale E. Gary ${ }^{1}$ \\ ${ }^{1}$ New Jersey Institute of Technology, Newark, NJ 07102, USA \\ ${ }^{2}$ Ioffe Institute, St. Petersburg 194021, Russia \\ email: gfleishm@njit.edu
}

\begin{abstract}
The coming years will see routine use of solar data of unprecedented spatial and spectral resolution, time cadence, and completeness in the wavelength domain. To capitalize on the soon to be available radio facilities such as the expanded OVSA, SSRT and FASR, and the challenges they present in the visualization and synthesis of the multi-frequency datasets, we propose that realistic, sophisticated 3D active region and flare modeling is timely now and will be a forefront of coronal studies over the coming years. Here we summarize our 3D modeling efforts, aimed at forward fitting of imaging spectroscopy data, and describe currently available 3D modeling tools. We also discuss plans for future generalization of our modeling tools.
\end{abstract}

Keywords. Sun: corona, Sun: magnetic fields, radiation mechanisms: non-thermal, methods: numerical, Sun: radio radiation, Sun: flares, stars: flares

\section{Introduction}

Solar activity, although energetically driven by subphotospheric processes, depends critically on coronal magnetism, which, broadly speaking, includes magnetic field generation, evolution, and transformation into kinetic, thermal, and nonthermal energies in the corona. Reliable tools for doing direct diagnostics have been lacking, although the situation is currently changing. Indeed, new space- and ground-based solar optical telescopes are already capable of precise measurements of the photospheric magnetic field with subarcsecond angular resolution and high temporal resolution. When combined with modern extrapolation algorithms, these data offer important clues on the coronal magnetic field structure and evolution. However, given the finite angular resolution, sensitivity, observational errors, and even theoretical limitations, such extrapolations are not unique, so the extrapolations require independent verification. An opportunity for quantitative verification will be available when the new generation of high-resolution solar-dedicated radio instruments (expanded OVSA, SSRT, and FASR) become operational. Microwave radiation is produced by the gyrosynchrotron (GS) mechanism as accelerated fast electrons gyrate in the coronal magnetic field. As has been recently proven using simulated microwave data, the coronal magnetic field can indeed in principle be reliably recovered at the flare dynamic time scales from the radio data, along with the key parameters of the thermal plasma and accelerated electrons (Fleishman et al. 2009). The ability to detect the magnetic field and its changes on dynamic time scales is a critically needed element to uncover the fundamental physics driving solar flares, eruptions, and activity.

\section{Modeling Methods and Tools}

Direct Modeling. 3D models of the solar flares are not yet numerous. Those available models (Preka-Papadema \& Alissandrakis 1992, Kucera et al. 1993, Bastian et al. 1998, 

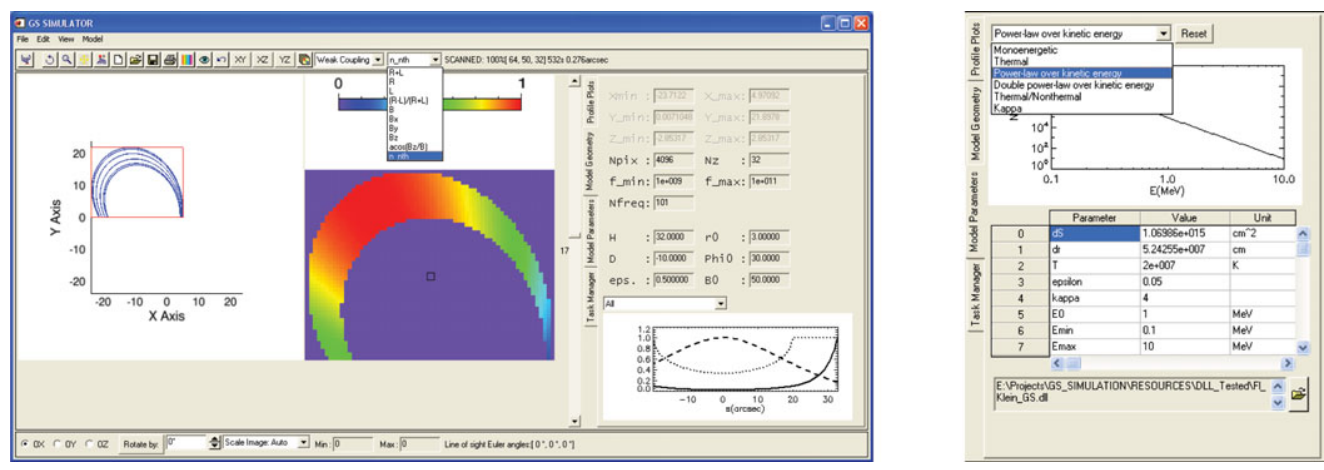

Figure 1. Left: interface of the model geometry and non-uniform parameter input-model magnetic loop (left window); nonuniform distribution of fast electrons given in a color code (middle window); and numeric input and graph display of the parameters involved. Right: selection of the fast electron energy distribution from a number of pre-defined analytical functions and parameters of this distribution.

Simões \& Costa 2006, 2010, Tzatzakis et al. 2008, Fleishman et al. 2009) are built on an idealized (e.g., dipole) magnetic loop, rather than a realistic magnetic geometry.

Our currently available modeling tool, GS Simulator, is also built based on an analytical (dipole) magnetic field model. A flaring loop model (i.e., user specified dipole flux tube, Figure 1, left) is produced with a newly developed interactive IDL widget application intended to provide a flexible tool that allows the user to generate spatially resolved GS spectra. To do so, the user populates the loop with thermal plasma by selecting plasma temperature and density in the adjustable parameter list, Figure 1, right, and specifies a fast electron population by selecting one of a few pre-defined distributions of the electrons over energy and pitch-angle and choosing numeric parameters for these distribution (Figure 1, right). The spatial distribution of the electrons is also specified along the loop, as shown in the color coded image (Figure 1, left). After selecting the angle from which to view the loop with the mouse, the tool then calculates the physical parameters along each line of sight needed to solve the radiation transfer equation, which is performed by external callable computing blocks. The default codes generating the GS (and free-free) emission based on the input geometrical line-of-sight model data were written in FORTRAN and $\mathrm{C}++$ based on fast GS codes newly developed by ?, and compiled as a DLL (or SO in case of Linux) callable by IDL.

The object-based architecture of this application provides the user with full 3D interaction with a predefined, but adjustable, magnetic loop geometry, as well as with any user defined analytical geometrical model that would inherit the basic properties of the generic "gs_model" IDL object defined in this package. To allow for more realistic 3D flare modeling, in place of drawing a loop "by hand", the tool must be further developed to include (i) a numerical magnetic field structure, such as would be obtained from a photospheric extrapolation (Figure 2) or a full MHD model based on vector photospheric measurements of the magnetic and velocity fields, along with the thermal plasma distribution, (ii) a realistic electron acceleration and transport model, and (iii) the ability to quickly compute emission in various wavelength regimes. The tool will enable the user to identify, from the model or through comparison with observations, the subset of field lines involved in flaring, consider fast electron transport in this realistic magnetic structure, calculate radiation from this evolving volume, and so simulate a solar flare.

Forward Fitting. The coronal magnetic field is a key parameter controlling most solar flaring activity, particle acceleration and transport. It has been understood, and 

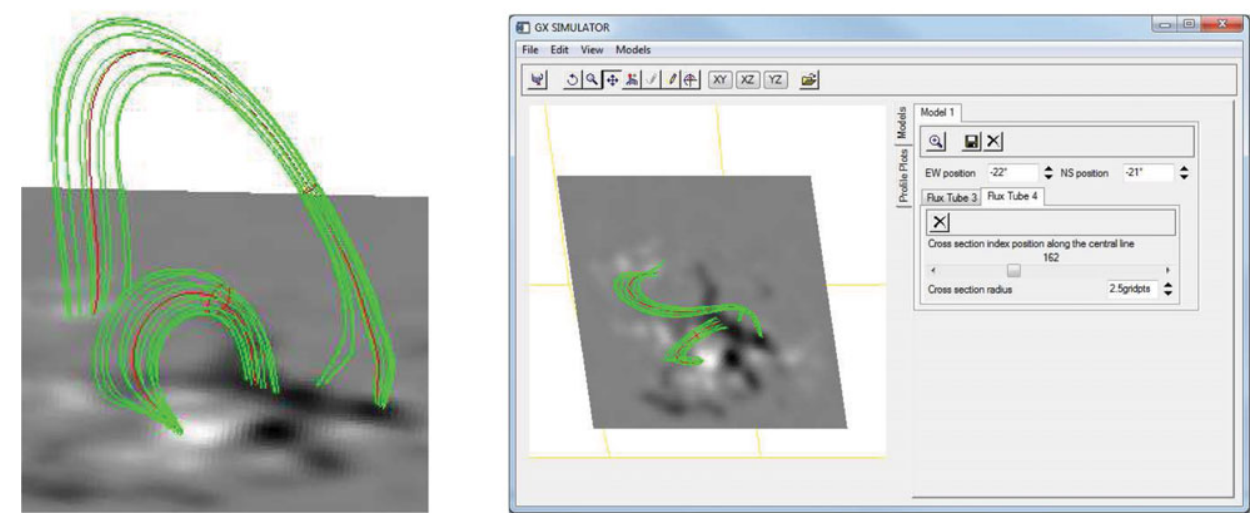

Figure 2. Left: Two flux tube models. They can be defined independently, and may represent interacting loops. Additional controls are being prepared for populating the loops with particles and ambient medium. Even dynamics of the particles is planned. Right: The loops now viewed from a specified location on the solar disk (22 E $21 \mathrm{~S}$ in this example). The tabs labeled Flux Tube 3 and Flux Tube 4 represent the two loops.

often proposed, that the coronal magnetic field along with fast electron distribution can in principle be evaluated from the microwave GS radiation, which is indeed sensitive to the instantaneous magnetic field strength and orientation relative to the line of sight and to the fast electron spectrum.

Diagnostics, understood as the determination of physical parameters of a system under study from arrays of observed parameters, is a key outstanding problem in Solar Physics. In some (basically linear) cases regularized true inversions can work well (e.g., Kontar et al. 2004). In most of the cases, however, such true inversions fail because of the highly nonlinear nature of physical systems. In such cases, the forward fitting, i.e., finding a number of free parameters of a physically motivated model of the system from fitting the model to observations, can often be used successfully in place of true inversions.

Anticipating a large breakthrough in the radio imaging spectroscopy observations, which will become possible soon due to the next generation of radio instruments, we have developed a practical forward fitting method, based on the SIMPLEX algorithm with shaking, that allows reliable derivation of the magnetic field and other parameters along a solar flaring loop using microwave imaging spectroscopy of GS emission, which is calculated with newly developed fast GS codes (Fleishman \& Kuznetsov 2010). We illustrate the method using a model loop with spatially varying magnetic field, filled with uniform ambient density and an evenly distributed fast electron population with an isotropic, power-law energy distribution (Fleishman et al. 2009).

From the flare radio model (Figure 3, left) described above we have a sequence of spatially resolved microwave spectra (in a general case, both intensity and polarization data, one spectrum per pixel). Then, we fit the data to a model microwave spectrum pixel by pixel (Figure 3, middle) to derive physical parameters of the source (e.g., the magnetic field, Figure 3, right). Although the exact GS formulae are very computationally expensive, much faster codes giving the same accuracy have recently been developed by Fleishmann and Kuznetsov (2010), which are used in practice as the forward fitting input. Then, having a fitting procedure resulting in fast and reliable finding of the true source parameters is exceedingly important. The problem here is that most of the minimization algorithms often find a local minimum of the normalized residual (or of the reduced chisquare), while the ultimate goal of the fitting is to identify the global minimum. So far, 

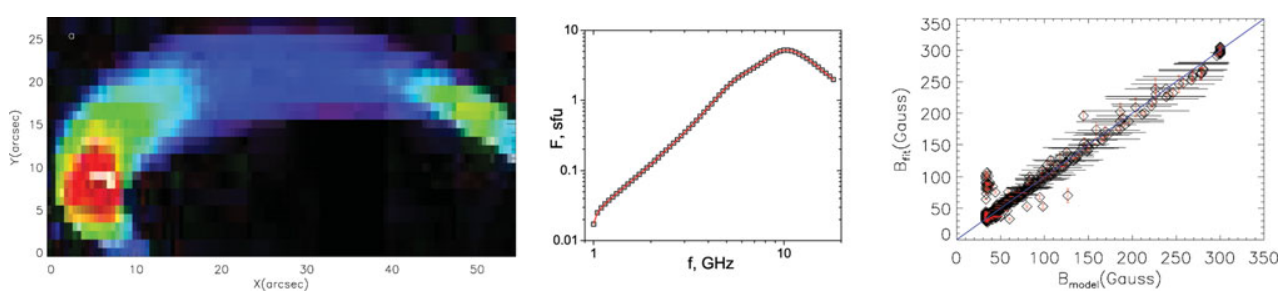

Figure 3. Simulated image of the radio emitting loop source at $4 \mathrm{GHz}$ as observed by an ideal radio heliograph with $\sim 1$ " pixel size resolution (left). Example of the model (symbols) and fit (solid curve) spectra corresponding to one particular pixel of the image displayed in the left panel (middle). Model-to-fit comparison of the magnetic field (right).

we determined that the simplex algorithm is very efficient in finding a local minimum. Then, it needs to be 'shaken' for the simplex solution to overcome any local minima and continue downhill towards the global minimum (a version of the stimulated annealing approach). Even when the algorithm performance is overall good, there is a non-zero probability that the algorithm fails to find the true solution in some pixels. We use post-processing to identify and flag/remove those pixels.

\section{Conclusions}

Modeling flare geometry, its full 3D visualization, and interactive adjustments to the user-specific needs are highly complicated tasks in themselves. Our modeling tools offer a united solution for these problems, which are widely applicable to various external data cube inputs and so offer a convenient framework for diverse studies of coronal magnetism, including flares and the active region magnetosphere. The modeling tools, computational libraries, and documentation are available via author's web page, see http://web.njit.edu/ gfleishm/.

The outlined modeling efforts can only bring fundamental knowledge about flare/active region physics if used in conjunction with modern, high-resolution observations. Key observations of the coronal plasma parameters can only be made by radio instruments that combine high sensitivity, temporal, spatial, and spectral resolution, which are unavailable now. A small part of the required science will be possible soon with the expanded OVSA instrument (anticipated operation of the upgraded instrument begins in fall, 2013) and the upgraded multi-wavelength SSRT. However, the full required capability has to wait until the full FASR (Gary 2003) has been built.

\section{Acknowledgments}

This work was supported in part by NSF grants ATM-0707319, AST-0908344, and AGS-0961867 and NASA grant NNX10AF27G to New Jersey Institute of Technology, and by the RFBR grants No. 08-02-92228, 09-02-00226, 09-02-00624. 